\title{
Steady-State Resonant Sloshing in an Upright Cylindrical Container Performing a Circular Orbital Motion
}

\author{
Ihor Raynovskyy $\mathbb{D}^{1}$ and Alexander Timokha $\mathbb{i}^{1,2}$ \\ ${ }^{1}$ Institute of Mathematics, National Academy of Sciences of Ukraine, Tereschenkivska 3 str., Kiev 01004, Ukraine \\ ${ }^{2}$ Centre for Autonomous Marine Operations and Systems, Department of Marine Technology, Norwegian University of \\ Science and Technology, 7491 Trondheim, Norway
}

Correspondence should be addressed to Alexander Timokha; atimokha@gmail.com

Received 14 January 2018; Accepted 5 June 2018; Published 28 June 2018

Academic Editor: R Aguilar-López

Copyright (c) 2018 Ihor Raynovskyy and Alexander Timokha. This is an open access article distributed under the Creative Commons Attribution License, which permits unrestricted use, distribution, and reproduction in any medium, provided the original work is properly cited.

\begin{abstract}
The nonlinear Narimanov-Moiseev multimodal equations are used to study the swirling-type resonant sloshing in a circular base container occurring due to an orbital (rotary) tank motion in the horizontal plane with the forcing frequency close to the lowest natural sloshing frequency. An asymptotic steady-state solution is constructed and the response amplitude curves are analyzed to prove their hard-spring type behavior for the finite liquid depth (the mean liquid depth-to-the-radius ratio $h>1$ ). This behavior type is supported by the existing experimental data. The wave elevations at the vertical wall are satisfactorily predicted except for a frequency range where the model test observations reported wave breaking and/or mean rotational flows.
\end{abstract}

\section{Introduction}

Caused by spacecraft and offshore applications (see chap. 1 in [1]), the liquid sloshing dynamics in vertical cylindrical tanks have been in focus for analytical, experimental, and numerical studies starting from the 50s. The earlier studies are outlined in [2-6], reviews of recent analytical and experimental results can be found in [5-9], and the CFD methods are described in [10] and [1, chap. 10]. Analytical studies of resonant liquid sloshing are normally used to classify the steady-state waves (frequency-domain problem), but CFD solvers simulate transient waves and hydrodynamic loads with different initial scenarios (time-domain problem). The contemporary solvers are typically based on viscous hydrodynamic models but the analytical methods (for clean tanks) assume an inviscid incompressible liquid with irrotational flows. In spite of that simplification, the methods provide a rather accurate prediction (chaps. 8-9 of [1] and $[6,11-13]$ ) of wave elevation as well as resulting hydrodynamic force and moment, at least, for the finite liquid depth.
One of the oldest frequency-domain problems on nonlinear sloshing in an upright circular cylindrical container consists of classifying the steady-state wave regimes due to the longitudinal harmonic forcing with the forcing frequency close to the lowest natural sloshing frequency. The problem was stated and solved, in the context of spacecraft applications, experimentally $[2-5,14]$ and theoretically $[5$, $6,11-13]$. For this forcing type, the theory establishes the planar steady-state standing wave and the so-called swirling wave mode (azimuthal progressive waves); it also detects a frequency range where these two steady-state wave regimes are not stable and irregular (chaotic) waves are expected. The theoretical results on effective frequency ranges of the steady-state wave regimes, wave elevations, and hydrodynamic forces, which were obtained by using the nonlinear (asymptotic) Narimanov-Moiseev multimodal theory [6], are in a satisfactory agreement with experimental data [2-4]. Along with quantifying the steady-state wave sloshing for the longitudinal forcing, the Narimanov-Moiseev multimodal theory [6] was also used for classifying the wave regimes due to elliptic-type tank excitations with a particular emphasis 
on the orbital (rotary) forcing. However, [6] did not either establish a link with actual applications, where these threedimensional forcing types appear, or validate the obtained mathematical results by experimental data. Bearing in mind sloshing in bioreactor [15-19], the present paper modifies the Narimanov-Moiseev theory to study swirling-type resonant waves due to the rotary forcing.

Ludwig von Prandtl [20] was, most probably, the first one who experimentally studied the orbitally excited swirling and described several accompanying free-surface phenomena, among which he pointed out (i) wave breaking and (ii) steady liquid rotation (henceforth, Prandtl mean mass-transport). The phenomena (i) and (ii) were experimentally observed in [7, 14-19]. Practical interests in bioreactors [15-19] recently motivated some authors to conduct more systematic model tests $[16,17,19]$. They also obtained a theoretical prediction $[15,18]$ using the linear sloshing theory (see chap. 5 in [1]). The theory fails at the primary resonance zone where it gives infinite wave amplitude but experiments [15-19] exhibit the hard-spring type wave-amplitude response. An integral effect of the wave breaking phenomenon may be accounted for by introducing the associated damping [21, 22]. Attempts to explain the Prandtl mass-transport by the azimuthal Stokes drift (see sect. 9.6.3 in [1]) were successful for the nonresonant frequencies but, as noted in $[14,20]$, this is not the case, if sloshing exhibits strongly nonlinear character; [2] suggested that the Prandtl mass-transport may be associated with the constant- $\mathrm{V}$ vortex whose explanation requires a vortical hydrodynamic model.

In summary, modeling the resonant liquid sloshing dynamics in a circular base container exposed to the orbital rotary forcing requires accounting for the free-surface nonlinearity, damping, and, when the Prandtl mass-transport matters, the vorticity.

In the present paper, to analytically describe the rotaryexcited damped nonlinear resonant sloshing, the NarimanovMoiseev modal equations from [6] are equipped with the linear damping terms. The damping coefficients are not limited to account for the boundary layer effect [23] but rather imply a cumulative effect by different dissipative phenomena including the wave breaking. The vorticity is not included into the mathematical model. The steady-state asymptotic solution of the modified Narimanov-Moiseev modal equations is constructed. This solution, as we will show, implies the swirling wave mode whose azimuthal propagation coincides with the rotary forcing direction. The analytics states the hard-spring type wave-amplitude response when the nondimensional (scaled by the tank radius) liquid depth $h>1$. This qualitative result is supported by experiments. The measured wave magnitude $[15,18]$ at the vertical wall is compared with its theoretical prediction. A good agreement is found for the frequency ranges where $[15,18]$ reported that the phenomena (i) and (ii) may be neglected. An important fact is that any speculations with increasing/decreasing the damping coefficients in the mathematical model cannot help matching the measured values in the frequency range where (i) and (ii) matter. This means that a further modification of the mathematical (hydrodynamic) model should consist in accounting for the vorticity.

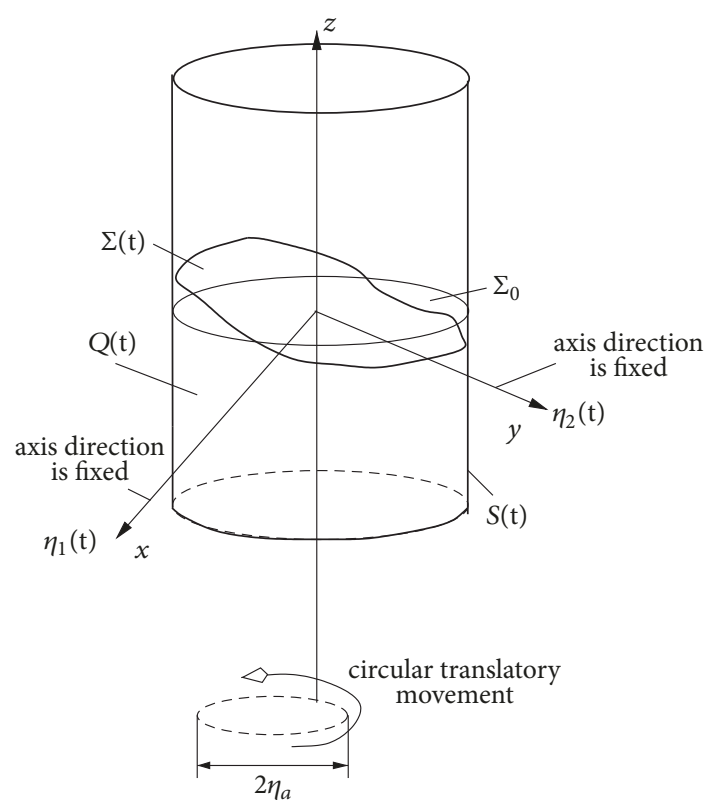

FIGURE 1: A sketch of a rigid upright circular cylindrical container moving translatory in the horizontal plane along a circular trajectory of the nondimensional radius $\eta_{a}$. The time-dependent liquid domain $Q(t)$ is confined by the free surface $\Sigma(t)$ ( $\Sigma_{0}$ is the mean liquid surface) and $S(t)$ is the wetted tank surface. Sloshing is considered in the tank-fixed coordinate system Oxyz.

\section{The Modified Narimanov-Moiseev Multimodal Theory}

The resonant liquid sloshing in a vertical circular cylindrical container of radius $r_{0}$ is considered in the tank-fixed (cylindrical) coordinate system. The problem is studied in the nondimensional statement assuming that $r_{0}$ is the characteristic tank size and $1 / \sigma$ ( $\sigma$ is the forcing frequency) is the characteristic time. The container performs a smallamplitude translatory orbital counterclockwise (without loss of generality) motion described by the two nondimensional generalized coordinates $\eta_{1}(t)=\eta_{a} \cos t$ and $\eta_{2}(t)=\eta_{a} \sin t$, where the forcing amplitude is small, $\eta_{a}=O(\varepsilon) \ll 1$.

Following [6], an inviscid incompressible liquid with irrotational flows is assumed. Figure 1 introduces geometric notations and explains how the tank axis moves along a circular trajectory of radius $\eta_{a}$. The free surface is governed by the single-valued representation $z=\zeta(r, \theta, t)$ (in the cylindrical coordinate system) and the liquid flow is determined by the velocity potential $\Phi(r, \theta, z, t)$. The unknowns, $\zeta$ and $\Phi$, are defined in the tank-fixed coordinate system Oxyz.

The functions $\zeta$ and $\Phi$ can be found from either the corresponding free-surface problem or its Bateman-Luke variational formulation (see chap. 7 of [1]). The latter variational formulation facilitates the nonlinear multimodal method that is based on the Fourier-type representations of $\zeta$ and $\Phi$ by the natural sloshing modes, which come from the spectral boundary problem

$$
\nabla^{2} \varphi_{M i}=0 \quad \text { in } Q_{0}
$$




$$
\begin{aligned}
\frac{\partial \varphi_{M i}}{\partial n} & =0 \quad \text { on } S_{0} ; \\
\frac{\partial \varphi_{M i}}{\partial z} & =\kappa_{M i} \varphi_{M i} \quad \text { on } \Sigma_{0} ; \\
\int_{\Sigma_{0}} \varphi_{M i} d S & =0
\end{aligned}
$$

where $\kappa_{M i}$ are the eigenvalues, $\Sigma_{0}$ is the mean (unperturbed) free surface, $Q_{0}$ is the mean liquid domain, and $S_{0}$ is the mean wetted tank surface. The spectral problem (1) has the analytical solution in the cylindrical coordinate system

$$
\begin{aligned}
\varphi_{M i}(r, \theta, z) & =\alpha_{M i} J_{M}\left(k_{M i} r\right) Z_{M i}(z)\left[\frac{\sin }{\cos }\right](M \theta) ; \\
M \geq 0, i>1 & \\
Z_{M i}(z) & =\frac{\cosh \left(k_{M i}(z+h)\right)}{\cosh \left(k_{M i} h\right)} ; \\
\kappa_{M i} & =k_{M i} \tanh \left(k_{M i} h\right),
\end{aligned}
$$

where $J_{M}^{\prime}\left(k_{M i}\right)=0, h$ is the nondimensional mean liquid depth, and $\alpha_{M i}$ are the normalizing coefficients $\alpha_{M i}^{-2}=$ $\int_{0}^{1} r J_{M}^{2}\left(k_{M i} r\right) \mathrm{d} r$; the dimensional natural sloshing frequencies are

$$
\begin{aligned}
\sigma_{M i} & =\sqrt{\kappa_{M i} \bar{g}} ; \\
\bar{g} & =\frac{g}{R_{0}} ; \\
\bar{\sigma}_{M i} & =\frac{\sigma_{M i}}{\sigma},
\end{aligned}
$$

and $g$ is the gravity acceleration.

Based on the fully nonlinear modal system from chap. 7 of [1], [6] derived the Narimanov-Moiseev modal equations adopting the Fourier-type solutions

$$
\begin{aligned}
& \Phi(r, \theta, z, t)=\dot{\eta}_{1}(t) r \cos \theta+\dot{\eta}_{2}(t) r \sin \theta \\
& \quad+\sum_{M i} \alpha_{M i} J_{M}\left(k_{M i} z\right) \\
& \cdot\left[P_{M i}(t) \cos (M \theta)+R_{m i}(t) \sin (m \theta)\right],
\end{aligned}
$$

where $P_{M i}(t)$ and $R_{m i}(t)$ are the generalized velocities and

$$
\begin{aligned}
z= & \zeta(r, \theta, t)=\sum_{M i} \alpha_{M i} J_{M}\left(k_{M i} r\right) \\
& \cdot\left(p_{M i}(t) \cos (M \theta)+r_{m i}(t) \sin (m \theta)\right),
\end{aligned}
$$

in which $p_{M i}(t)$ and $r_{m i}(t)$ are the sloshing-related generalized coordinates.

The derivations assume that the forcing frequency $\sigma$ is close to the lowest natural sloshing frequency $\sigma_{1}=\sigma_{11}$ and there are no secondary resonances (see what the secondary resonance means in chaps. 8 and 9 of [1]). The NarimanovMoiseev multimodal theory suggests that the resonant wave response is of the order $O\left(\varepsilon^{1 / 3}\right)$ and the dominant asymptotic contribution is associated with the lowest natural sloshing modes $\left(p_{11}\right.$ and $\left.r_{11}\right)$. Due to the trigonometry by the angular coordinate $\theta$ (see [6]),

$$
\begin{aligned}
p_{11} & \sim r_{11}=O\left(\varepsilon^{1 / 3}\right) ; \\
p_{0 j} & \sim p_{2 j} \sim r_{2 j}=O\left(\varepsilon^{2 / 3}\right) ; \\
r_{1(j+1)} & \sim p_{1(j+1)} \sim r_{3 j} \sim p_{3 j}=O(\varepsilon),
\end{aligned}
$$

$$
j \geq 1 ;
$$

the remaining generalized coordinates are of the order $o(\varepsilon)$. When neglecting the $o(\varepsilon)$-quantities, the modal equations (with respect to $p_{M i}$ and $r_{m i}$ ) take the following form [6]:

$$
\begin{aligned}
& \ddot{p}_{11}+2 \xi_{11} \bar{\sigma}_{11} \dot{p}_{11}+\bar{\sigma}_{11}^{2} p_{11}+d_{1} p_{11}\left(\ddot{p}_{11} p_{11}+\ddot{r}_{11} r_{11}\right. \\
& \left.+\dot{p}_{11}^{2}+\dot{r}_{11}^{2}\right)+d_{2}\left[r_{11}\left(\ddot{p}_{11} r_{11}-\ddot{r}_{11} p_{11}\right)\right. \\
& \left.+2 \dot{r}_{11}\left(\dot{p}_{11} r_{11}-\dot{r}_{11} p_{11}\right)\right] \\
& +\sum_{j=1}^{\infty}\left[d_{3}^{(j)}\left(\ddot{p}_{11} p_{2 j}+\ddot{r}_{11} r_{2 j}+\dot{p}_{11} \dot{p}_{2 j}+\dot{r}_{11} \dot{r}_{2 j}\right)\right. \\
& +d_{4}^{(j)}\left(\ddot{p}_{2 j} r_{11}+\ddot{r}_{2 j} r_{11}\right)+d_{5}^{(j)}\left(\ddot{p}_{11} p_{0 j}+\dot{p}_{11} \dot{p}_{0 j}\right) \\
& \left.+d_{6}^{(j)} \ddot{p}_{0 j} p_{11}\right]=-\ddot{\eta}_{1} \kappa_{11} P_{1}, \\
& \ddot{r}_{11}+2 \xi_{11} \bar{\sigma}_{11} \dot{r}_{11}+\bar{\sigma}_{11}^{2} r_{11}+d_{1} r_{11}\left(\ddot{p}_{11} p_{11}+\ddot{r}_{11} r_{11}\right. \\
& \left.+\dot{p}_{11}^{2}+\dot{r}_{11}^{2}\right)+d_{2}\left[p_{11}\left(\ddot{r}_{11} p_{11}-\ddot{p}_{11} r_{11}\right)\right. \\
& \left.+2 \dot{p}_{11}\left(\dot{r}_{11} p_{11}-\dot{p}_{11} r_{11}\right)\right] \\
& +\sum_{j=1}^{\infty}\left[d_{3}^{(j)}\left(\ddot{p}_{11} r_{2 j}-\ddot{r}_{11} p_{2 j}+\dot{p}_{11} \dot{r}_{2 j}-\dot{p}_{2 j} \dot{r}_{11}\right)\right. \\
& +d_{4}^{(j)}\left(\ddot{r}_{2 j} p_{11}-\ddot{p}_{2 j} r_{11}\right)+d_{5}^{(j)}\left(\ddot{r}_{11} p_{0 j}+\dot{r}_{11} \dot{p}_{0 j}\right) \\
& \left.+d_{6}^{(j)} \ddot{p}_{0 j} r_{11}\right]=-\ddot{\eta}_{2} \kappa_{11} P_{1} ; \\
& \ddot{p}_{2 k}+2 \xi_{2 k} \bar{\sigma}_{2 k} \dot{p}_{2 k}+\bar{\sigma}_{2 k}^{2} p_{2 k}+d_{7, k}\left(\dot{p}_{11}^{2}-\dot{r}_{11}^{2}\right) \\
& +d_{9 k}\left(\ddot{p}_{11} p_{11}-\ddot{r}_{11} r_{11}\right)=0, \quad k \geq 1 ; \\
& \ddot{r}_{2 k}+2 \xi_{2 k} \bar{\sigma}_{2 k} \dot{r}_{2 k}+\bar{\sigma}_{2 k}^{2} r_{2 k}+2 d_{7, k} \dot{p}_{11} \dot{r}_{11} \\
& +d_{9, k}\left(\ddot{p}_{11} r_{11}+\ddot{r}_{11} p_{11}\right)=0, \quad k \geq 1 ; \\
& \ddot{p}_{0 k}+2 \xi_{2 k} \bar{\sigma}_{2 k} \dot{p}_{2 k}+\bar{\sigma}_{0 k}^{2} p_{0 k}+d_{8, k}\left(\dot{p}_{11}^{2}+\dot{r}_{11}^{2}\right) \\
& +d_{10, k}\left(\ddot{p}_{11} p_{11}+\ddot{r}_{11} r_{11}\right)=0, \quad k \geq 1 ;
\end{aligned}
$$




$$
\begin{aligned}
& \ddot{p}_{3 k}+2 \xi_{3 k} \bar{\sigma}_{3 k} \dot{p}_{3 k}+\bar{\sigma}_{3 k}^{2} p_{3 k}+d_{11, k}\left[\ddot{p}_{11}\left(p_{11}^{2}-r_{11}^{2}\right)\right. \\
& \left.-2 p_{11} r_{11} \ddot{r}_{11}\right]+d_{12, k}\left[p_{11}\left(\dot{p}_{11}^{2}-\dot{r}_{11}^{2}\right)\right. \\
& \left.-2 r_{11} \dot{p}_{11} \dot{r}_{11}\right]+\sum_{j=1}^{\infty}\left[d_{13, k}^{(j)}\left(\ddot{p}_{11} p_{2 j}-\ddot{r}_{11} r_{2 j}\right)\right. \\
& +d_{14, k}^{(j)}\left(\ddot{p}_{2 j} p_{11}-\ddot{r}_{2 j} r_{11}\right)+d_{15, k}^{(j)}\left(\dot{p}_{2 j} p_{11}-\dot{r}_{2 j} r_{11}\right) \\
& =0, \quad k \geq 1 \\
& \ddot{r}_{3 k}+2 \xi_{3 k} \bar{\sigma}_{3 k} \dot{r}_{3 k}+\bar{\sigma}_{3 k}^{2} r_{3 k}+d_{11, k}\left[\ddot{r}_{11}\left(p_{11}^{2}-r_{11}^{2}\right)\right. \\
& \left.+2 p_{11} r_{11} \ddot{p}_{11}\right]+d_{12, k}\left[r_{11}\left(\dot{p}_{11}^{2}-\dot{r}_{11}^{2}\right)\right. \\
& \left.+2 p_{11} \dot{p}_{11} \dot{r}_{11}\right]+\sum_{j=1}^{\infty}\left[d_{13, k}^{(j)}\left(\ddot{p}_{11} r_{2 j}+\ddot{r}_{11} p_{2 j}\right)\right. \\
& \left.+d_{14, k}^{(j)}\left(\ddot{p}_{2 j} r_{11}+\ddot{r}_{2 j} p_{11}\right)+d_{15, k}^{(j)}\left(\dot{p}_{2 j} \dot{r}_{11}+\dot{r}_{2 j} \dot{p}_{11}\right)\right] \\
& =0, \quad k \geq 1 \\
& \ddot{p}_{1 n}+2 \xi_{1 n} \bar{\sigma}_{1 n} \dot{p}_{1 n}+\bar{\sigma}_{1 n}^{2} p_{1 n}+d_{16, n}\left(\ddot{p}_{11} p_{11}^{2}\right. \\
& \left.+r_{11} p_{11} \ddot{r}_{11}\right)+d_{17, n}\left(\ddot{p}_{11} r_{11}^{2}-r_{11} p_{11} \ddot{r}_{11}\right) \\
& +d_{18, n} p_{11}\left(\dot{p}_{11}^{2}+\dot{r}_{11}^{2}\right)+d_{19, n}\left(r_{11} \dot{p}_{11} \dot{r}_{11}-p_{11} \dot{r}_{11}^{2}\right) \\
& +\sum_{j=1}^{\infty}\left[d_{20, n}^{(j)}\left(\ddot{p}_{11} p_{2 j}+\ddot{r}_{11} r_{2 j}\right)\right. \\
& +d_{21, n}^{(j)}\left(p_{11} \ddot{p}_{2 j}+\ddot{r}_{11} r_{2 j}\right)+d_{22, n}^{(j)}\left(\dot{p}_{11} \dot{p}_{2 j}+\dot{r}_{11} r_{2 j}\right) \\
& \left.+d_{23, n}^{(j)} \ddot{p}_{11} p_{0 j}+d_{24, n}^{(j)} p_{11} \ddot{p}_{0 j}+d_{25, n}^{(j)} \dot{p}_{11} \dot{p}_{0 j}\right] \\
& =-\ddot{\eta}_{1} \kappa_{1 n} P_{n}, \quad n \geq 2, \\
& \ddot{r}_{1 n}+2 \xi_{1 n} \bar{\sigma}_{1 n} \dot{r}_{1 n}+\sigma_{1 n}^{2} r_{1 n}+d_{16, n}\left(\ddot{r}_{11} r_{11}^{2}\right. \\
& \left.+r_{11} p_{11} \ddot{p}_{11}\right)+d_{17, n}\left(\ddot{r}_{11} p_{11}^{2}-r_{11} p_{11} \ddot{p}_{11}\right) \\
& +d_{18, n} r_{11}\left(\dot{p}_{11}^{2}+\dot{r}_{11}^{2}\right)+d_{19, n}\left(p_{11} \dot{p}_{11} \dot{r}_{11}-r_{11} \dot{p}_{11}^{2}\right) \\
& +\sum_{j=1}^{\infty}\left[d_{20, n}^{(j)}\left(\ddot{p}_{11} p_{2 j}-\ddot{r}_{11} p_{2 j}\right)\right. \\
& +d_{21, n}^{(j)}\left(p_{11} \ddot{r}_{2 j}-r_{11} \ddot{p}_{2 j}\right)+d_{22, n}^{(j)}\left(\dot{p}_{11} \dot{r}_{2 j}-\dot{r}_{11} \dot{p}_{2 j}\right) \\
& \left.+d_{23, n}^{(j)} \ddot{r}_{11} p_{0 j}+d_{24, n}^{(j)} r_{11} \ddot{p}_{0 j}+d_{25, n}^{(j)} \dot{r}_{11} \dot{p}_{0 j}\right] \\
& =-\ddot{\eta}_{2} \kappa_{1 n} P_{n}, \quad n \geq 2,
\end{aligned}
$$

$\delta=\mathrm{Ga}^{-1 / 4}=\sqrt{\nu /\left(g^{1 / 2} R_{0}^{3 / 2}\right)} \ll 1$ (Ga is the Galilei number), and

$$
\begin{aligned}
& \mu_{M i}^{(0)}=\int_{0}^{1} r J_{M}^{2}\left(k_{M i} r\right) \mathrm{d} r ; \\
& \mu_{M i}^{(1)}=\int_{0}^{1} r k_{M i}^{2} J_{M}^{\prime 2}\left(k_{M i} r\right) \mathrm{d} r+M^{2} \int_{0}^{1} \frac{J_{M}^{2}\left(k_{M i} r\right)}{r} \mathrm{~d} r \\
& \mu_{M i}^{(2)}=M^{2}\left(\frac{\tanh \left(k_{M i} h\right)}{k_{M i}}+\frac{h}{\cosh ^{2}\left(k_{M i} h\right)}\right) ; \\
& \mu_{M i}^{(3)}=k_{M i}^{2}\left(\frac{\tanh \left(k_{M i} h\right)}{k_{M i}}-\frac{h}{\cosh ^{2}\left(k_{M i} h\right)}\right) .
\end{aligned}
$$

\section{Steady-State Asymptotic Solution}

The third-order sloshing-related generalized coordinates, which are governed by (12)-(15), are "driven" and, as a consequence, as long as we know an analytical solution of (7)-(11) (in which only first two equations have inhomogeneous righthand sides), one can easily get an analytical solution of (12)-(15), which becomes then a set of independent linear oscillators with known right-hand sides. The forthcoming analysis can therefore be restricted to (12)-(15).

To find an asymptotic periodic solution of (7)-(15), we pose the lowest-order generalized coordinates as follows:

$$
\begin{aligned}
& p_{11}(t)=a \cos (\sigma t)+\bar{a} \sin (\sigma t)+o\left(\varepsilon^{1 / 3}\right), \\
& r_{11}(t)=\bar{b} \cos (\sigma t)+b \sin (\sigma t)+o\left(\varepsilon^{1 / 3}\right),
\end{aligned}
$$


where $a, \bar{a}, b$, and $\bar{b}$ are the nondimensional amplitude parameters of the order $O\left(\varepsilon^{1 / 3}\right)$. Inserting (19) into (9)-(11) makes it possible to find the following asymptotic expressions:

$$
\begin{aligned}
& p_{0 k}(t)=s_{0 k}\left(a^{2}+\bar{a}^{2}+b^{2}+\bar{b}^{2}\right) \\
& +s_{1 k}\left[\left(a^{2}-\bar{a}^{2}-b^{2}+\bar{b}^{2}\right) \cos 2 t\right. \\
& +2(a \bar{a}+b \bar{b}) \sin 2 t]+O\left(\xi_{0 k} \varepsilon^{2 / 3}\right), \\
& p_{2 k}(t)=c_{0 k}\left(a^{2}+\bar{a}^{2}-b^{2}-\bar{b}^{2}\right) \\
& +c_{1 k}\left[\left(a^{2}-\bar{a}^{2}+b^{2}-\bar{b}^{2}\right) \cos 2 t\right. \\
& +2(a \bar{a}-b \bar{b}) \sin 2 t]+O\left(\xi_{2 k} \varepsilon^{2 / 3}\right), \\
& r_{2 k}(t)=2 c_{0 k}(a \bar{b}+\bar{a} b)+2 c_{1 k}[(a \bar{b}+\bar{a} b) \cos 2 t \\
& +2(a b+\bar{a} \bar{b}) \sin 2 t]+O\left(\xi_{2 k} \varepsilon^{2 / 3}\right),
\end{aligned}
$$

where

$$
\begin{aligned}
& s_{0 k}=\frac{d_{10, k}-d_{8, k}}{2 \bar{\sigma}_{0 k}^{2}} ; \\
& s_{1 k}=\frac{d_{10, k}+d_{8, k}}{2\left(\bar{\sigma}_{0 k}^{2}-4\right)} ; \\
& c_{0 k}=\frac{d_{9, k}-d_{7, k}}{2 \bar{\sigma}_{2 k}^{2}} ; \\
& c_{1 k}=\frac{d_{9, k}+d_{7, k}}{2\left(\bar{\sigma}_{2 k}^{2}-4\right)} .
\end{aligned}
$$

When deriving (20), we accounted for (16) as well as $\left(\bar{\sigma}_{0 k}^{2}-\right.$ $4)=O(1)$ and $\left(\bar{\sigma}_{2 k}^{2}-4\right)=O(1)$ (there are no secondary resonances). The remaining terms of (20) are therefore of the order $O(\varepsilon)$. Analogously, one can get an asymptotic solution of (12)-(15) in terms of $a, \bar{a}, b$, and $\bar{b}$; the result coincides with formulas from Appendix of [6].

Substituting (19) and (20) into (7) and (8) and gathering the first Fourier harmonics lead to the secular system of nonlinear algebraic equations

$$
\begin{gathered}
a\left[\Lambda+m_{1}\left(a^{2}+\bar{a}^{2}+\bar{b}^{2}\right)+m_{3} b^{2}\right] \\
+\bar{a}\left(\left(m_{1}-m_{3}\right) b \bar{b}+\xi\right)=\varepsilon, \\
\bar{a}\left[\Lambda+m_{1}\left(a^{2}+\bar{a}^{2}+b^{2}\right)+m_{3} \bar{b}^{2}\right] \\
+a\left(\left(m_{1}-m_{3}\right) b \bar{b}-\xi\right)=0,
\end{gathered}
$$

$$
\begin{gathered}
\bar{b}\left[\Lambda+m_{1}\left(a^{2}+b^{2}+\bar{b}^{2}\right)+m_{3} \bar{a}^{2}\right] \\
+b\left(\left(m_{1}-m_{3}\right) \bar{a} a+\xi\right)=0, \\
b\left[\Lambda+m_{1}\left(b^{2}+\bar{a}^{2}+\bar{b}^{2}\right)+m_{3} a^{2}\right] \\
+\bar{b}\left(\left(m_{1}-m_{3}\right) \bar{a} a-\xi\right)=\varepsilon,
\end{gathered}
$$

which plays the role of the solvability condition. Here, $\Lambda=$ $\bar{\sigma}_{11}^{2}-1=O\left(\varepsilon^{2 / 3}\right)$ expresses the so-called Moiseev detuning; $\xi=$ $2 \xi_{11}, \varepsilon=\eta_{a} \kappa_{11} P_{1}$, and $m_{i}=O(1)$ are the analytically known functions of the hydrodynamic coefficients $[6,22]$. Using the Moiseev detuning in expressions for $m_{1}$ and $m_{3}$ makes them exclusively functions of $h$.

In summary, having known $a, \bar{a}, b$, and $\bar{b}$ from (22) makes it possible to find all the sloshing-related generalized coordinates within the $o(\varepsilon)$-order quantities. Using formulas from [22] facilitates studying the stability of the constructed steady-state solution via the multitiming scheme and the linear Lyapunov method.

\section{Alternative Forms of the Secular System (22)}

When the damping coefficient $\xi$ is not zero, the waveamplitude response is characterized by the phase lags $\psi$ and $\varphi$, which can be introduced as

$$
\begin{aligned}
& a=A \cos \psi, \\
& \bar{a}=A \sin \psi, \\
& \bar{b}=B \cos \varphi, \\
& b=B \sin \varphi .
\end{aligned}
$$

The secular system (22) can then be rewritten in the form

$$
\begin{aligned}
& 1: A\left[\Lambda+m_{1} A^{2}+\left(m_{3}-F\right) B^{2}\right]=\varepsilon \cos \psi, \\
& 2: B\left[\Lambda+m_{1} B^{2}+\left(m_{3}-F\right) A^{2}\right]=\varepsilon \sin \varphi, \\
& 3: A\left[D B^{2}+\xi\right]=\varepsilon \sin \psi, \\
& 4: B\left[D A^{2}-\xi\right]=\varepsilon \cos \varphi, \\
& F=\left(m_{3}-m_{1}\right) \cos ^{2} \alpha=\frac{m_{3}-m_{1}}{1+C^{2}}, \\
& D=C F, \\
& \alpha=\varphi-\psi, \\
& C=\tan \alpha .
\end{aligned}
$$

System (24)-(25) couples the dominant sloshing amplitudes $A$ and $B$ in the $O x$ and $O y$ directions, respectively, as well as the corresponding phase lags. 
To exclude $\psi$ and $\varphi$ from (24) and reduce it to three equations with respect to $A, B$, and $C$, we insert $\varphi=\psi+\alpha$ into the right-hand sides of 2,4 and substitute $\varepsilon \cos \psi, \varepsilon \sin \psi$ taken from 1,3 . The result is the following system of linear homogeneous equations:

$$
\begin{aligned}
& A\left[\cos \alpha\left(D B^{2}+\xi\right)\right. \\
& \left.\quad+\sin \alpha\left(\Lambda+m_{1} A^{2}+\left(m_{3}-F\right) B^{2}\right)\right]-B[\Lambda \\
& \left.\quad+m_{1} B^{2}+\left(m_{3}-F\right) A^{2}\right]=0 \\
& A\left[\cos \alpha\left(\Lambda+m_{1} A^{2}+\left(m_{3}-F\right) B^{2}\right)\right. \\
& \left.\quad-\sin \alpha\left(D B^{2}+\xi\right)\right]-B\left[D A^{2}-\xi\right]=0,
\end{aligned}
$$

with respect to $A$ and $B$. The system must have a nontrivial solution. This leads to the zero-determinant condition

$$
\begin{aligned}
F & {\left[\xi^{2}+\left(\Lambda+m_{1}\left(A^{2}+B^{2}\right)\right)^{2}\right] } \\
& =F\left[F C^{2} A^{2} B^{2}+\xi C\left(A^{2}-B^{2}\right)\right]\left(m_{3}-m_{1}\right),
\end{aligned}
$$

which does not contain the phase lags. Another two equations without the phase lags are

$$
\begin{aligned}
& \left.1]^{2}+3\right]^{2} \\
& =A^{2}\left(\left[\Lambda+m_{1} A^{2}+\left(m_{3}-F\right) B^{2}\right]^{2}+\left[C F B^{2}+\xi\right]^{2}\right) \\
& =\varepsilon^{2}, \\
& \left.2{ }^{2}+4\right]^{2} \\
& =B^{2}\left(\left[\Lambda+m_{1} B^{2}+\left(m_{3}-F\right) A^{2}\right]^{2}+\left[C F A^{2}-\xi\right]^{2}\right) \\
& =\varepsilon^{2} .
\end{aligned}
$$

\section{The Swirling Wave Mode, the Response Curves, and Comparison with Experiments}

Within the framework of our asymptotic theory, the modal representation (5) takes the form

$$
\begin{gathered}
\zeta(r, \theta, t)=J_{1}\left(k_{11} r\right)[(a \cos \theta+\bar{b} \sin \theta) \cos t \\
+(\bar{a} \cos \theta+b \sin \theta) \sin t]+o\left(\varepsilon^{1 / 3}\right),
\end{gathered}
$$

which determines the swirling wave mode [22] unless

$$
\begin{aligned}
& a b=\bar{a} \bar{b} \Longleftrightarrow \\
& C=0
\end{aligned}
$$

when a standing wave is expected (because of (28), $A>0$ and $B>0)$.
Assuming $C=0 \Longrightarrow F=m_{3}-m_{1} \& \xi^{2}+m_{1}\left(A^{2}+B^{2}\right)=0$ that is impossible for both equations in (28). Thus, we proved the following.

$\left(1^{0}\right)$ The orbital rotary forcing always leads to the swirlingtype wave mode.

Equation (27) admits $F=0(D=C F=0) \Longrightarrow C=$ $\infty \Longrightarrow \varphi=\psi \pm \pi / 2$. The last two equations of (24) deduce $C=+\infty, \varphi=\psi+\pi / 2 \& A=B$. This means the following.

$\left(2^{0}\right)$ The orbital rotary forcing leads to the swirling wave mode, whose lowest-order amplitudes are governed by

$$
A^{2}\left[\left(\Lambda+\left(m_{1}+m_{3}\right) A^{2}\right)^{2}+\xi^{2}\right]=\varepsilon^{2} ; \quad A=B>0,
$$

$\varphi=\psi+\pi / 2$, where the phase lag $\psi$ comes from

$$
A\left[\Lambda+\left(m_{1}+m_{3}\right) A^{2}\right]=\varepsilon \cos \psi
$$

$$
A \xi=\varepsilon \sin \psi
$$

The Narimanov-Moiseev multimodal theory is applicable in a small neighborhood of the primary resonance for $h>1$ [6]. Calculations show that

$$
\begin{aligned}
& m_{1}<0, \\
& m_{3}>0, \\
& m_{1}+m_{3}>0 \\
& \quad \text { as } h>1 .
\end{aligned}
$$

The last inequality implies that the response curves by (31) have the hard-spring type behavior. This theoretical conclusion is supported by experiments $[15,18]$.

The hard-spring type response curves by (31) are illustrated in Figure 2. It shows the hysteresis in the frequency range between $W$ and $S$. The frequency ratio $\left(\sigma / \sigma_{1}\right)_{*}$ of the point $S$ is determined by

$$
\left(\frac{\sigma}{\sigma_{1}}\right)_{*}=\left(1-\left(m_{1}+m_{3}\right)\left(\frac{\varepsilon}{\xi}\right)^{2}\right)^{-1 / 2} .
$$

This frequency ratio increases with $\varepsilon / \xi$ so that having known $\xi$ and $\varepsilon$ makes it possible to evaluate $S$, where a jump from the upper to lower branch must occur with increasing the forcing frequency $\sigma / \sigma_{1}$.

Adopting the model tests with a stepwise increase of the forcing frequency, which provide a path-following along $P_{1} S$ in Figure 2, $[15,18]$ measured the steady-state wave elevations at the wall for the two rigid circular base containers of the radii 72 and $287 \mathrm{~mm}$ filled with tap water $(h=1.04)$. Several forcing amplitudes were tested. Our theory requires that the forcing amplitude $\eta_{a}$ is small and $\sigma / \sigma_{1}$ is in a neighborhood of the unit. Three experimental series in $[15,18]$ were done with $\eta_{a}=0.01,0.02$, and 0.04 , for which the constructed asymptotic theory may be applicable.

Formulas (17) estimate the lower bound of the damping coefficient as $\xi \geq 0.031$ for the experimental tanks in $[15,18]$ 


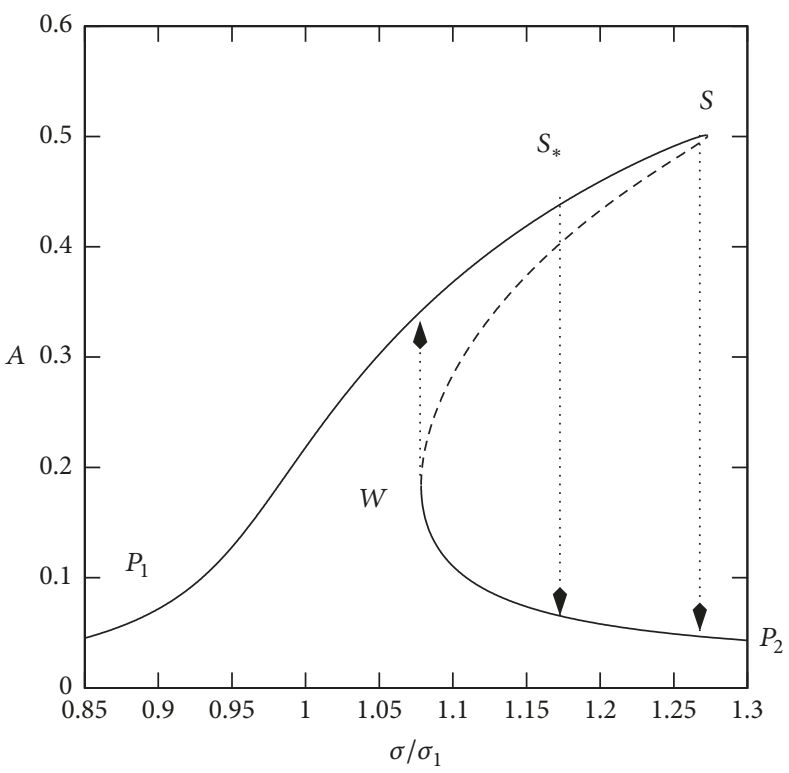

(a)

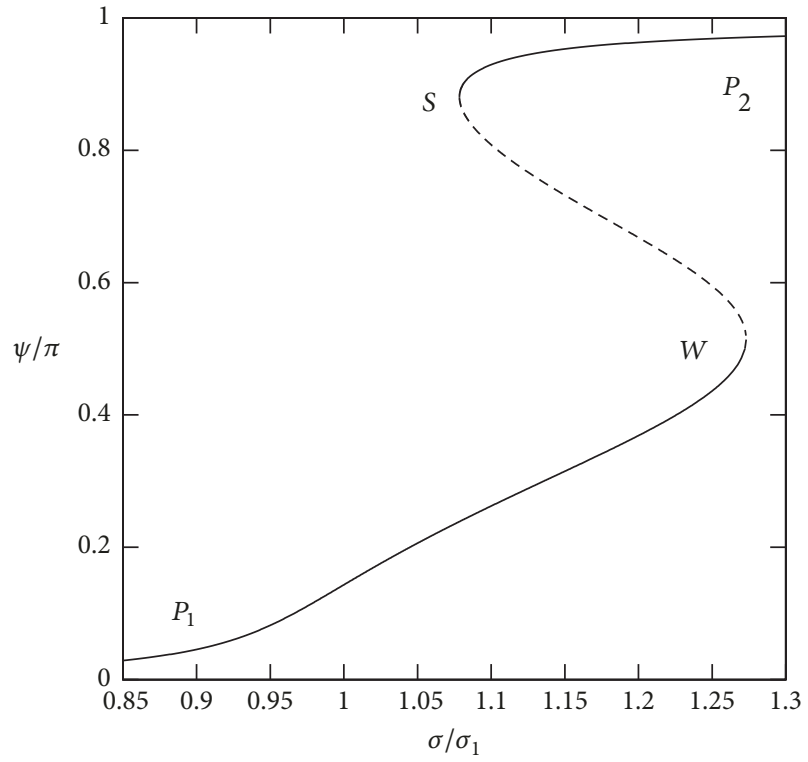

(b)

Figure 2: Theoretical response curves for the rotary forcing associated with an experimental case in [15]. The panel (a) shows the dominant wave amplitude but (b) corresponds to the phase lag versus the scaled forcing frequency. The solid lines imply the stability but the dashed lines mark the unstable steady-state sloshing. There is the hysteresis between $W$ and $S$, where two steady-state solutions coexist. Position of $S$ is determined by the damping rates. The calculations were done by using (31) and (32) with $\eta_{a}=0.02, h=1.04$, and $\xi=0.034$.

filled with tap water. Using the experimentally established frequencies where a jump from the upper to lower branch occurs (point $S$ in Figure 2), the formula (34) makes it possible to estimate the upper bound for the damping coefficient as $\xi \leq 0.034$ (for the forcing amplitudes 0.01 and 0.02 . $\left(\sigma / \sigma_{1}\right)_{*}=1.27$ and 1.45 , respectively). This means that damping in the model tests of $[15,18]$ is basically associated with the boundary layer and bulk viscosity by (17).

Adopting $\xi=0.034$ (the upper bound) in calculations with the constructed steady-state solution makes it possible to compare the theoretical wave elevations at the wall with the corresponding measurements in Figure 3. The figure demonstrates a good agreement for the empty symbols, where observations in $[15,18]$ did not demonstrate the wave phenomena (i) and (ii), which we described in Introduction. However, a strong discrepancy is found out for the filled symbols where these phenomena matter. Even though the wave breaking effect may often be modeled by increasing the damping rates [22], as we noted above, the experimental data require $\xi \leq 0.034$, and, therefore, getting a better agreement by increasing $\xi$ is theoretically impossible. Numerical tests confirmed that point.

\section{Conclusions}

Using the Narimanov-Moiseev multimodal sloshing theory [6], which describes the resonant sloshing in a circular base container due to the orbital forcing, we studied the steady-state wave response when the forcing frequency is close to the lowest natural sloshing frequency. An analytical asymptotic solution of the nonlinear modal equations is constructed when these equations are equipped with the linear damping terms. The constructed analytical solution shows that the wave-amplitude response has the hard-spring type behavior that is supported by experiments. Furthermore, the solution well describes wave elevations at the wall when the phenomena “(i), wave breaking” and “(ii), Prandtl's steady rotational flow" can be neglected according to observations in $[15,18]$. Even though the wave breaking effect may be successfully modeled by an increase of the damping rates, this approach is not applicable for the rotary forcing. To improve the analytical sloshing theory, one should include the Prandtl mass-transport phenomenon (the mean azimuthal rotation of the liquid column) into the mathematical model.

\section{Data Availability}

No data were used to support this study.

\section{Conflicts of Interest}

The authors declare that they have no conflicts of interest regarding the publication of this paper.

\section{Acknowledgments}

The second author acknowledges the financial support of the Centre of Autonomous Marine Operations and Systems (AMOS) whose main sponsor is the Norwegian Research Council (Project no. 223254-AMOS). 


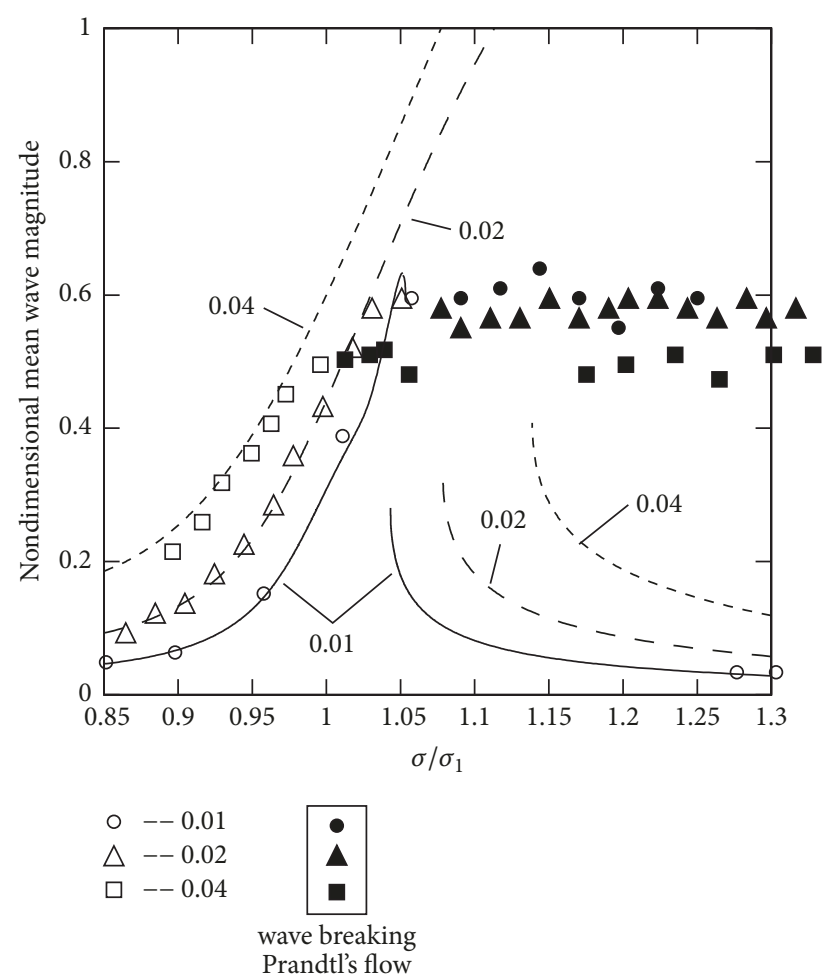

FIgURE 3: Theoretical (lines) and experimental (symbols) nondimensional wave magnitude at the vertical wall, $(1 / 2)\left(\zeta_{\max }-\zeta_{\min }\right)$, for the rotary forcing. Experiments $[15,18]$ were conducted by using a stepwise increase of the forcing frequency providing a pathfollowing along the upper branch in Figure 2. The theoretical curves were drawn by using the constructed lowest-order asymptotic analytical solution and formulas from Appendix of [6] for the higher-order generalized coordinates. The curves are marked by the nondimensional amplitudes $\eta_{a}=0.01,0.02$, and 0.04 . The computations adopted the damping coefficient $\xi=0.034$. The filled symbols mark the experimental cases when observations in $[15,18]$ detected both the strong wave breaking and the Prandtl phenomenon [20].

\section{References}

[1] O. M. Faltinsen and A. N. Timokha, Sloshing, Cambridge University Press, 2009.

[2] H. N. Abramson, W. H. Chu, and D. D. Kana, "Some studies of nonlinear lateral sloshing in rigid containers," Journal of Applied Mechanics, vol. 33, no. 4, pp. 777-784, 1964.

[3] R. E. Hutton, "An investigation of nonlinear, nonplanar oscillations of fluid in cylindrical container," Tech. Rep., 1963.

[4] H. N. Abramson, "The dynamic behavior of liquids in moving containers with applications to space vehicle technology," Tech. Rep., 1966.

[5] I. A. Lukovsky, Nonlinear Dynamics, vol. 27 of De Gruyter Studies in Mathematical Physics, De Gruyter, Berlin, Russian edition, 2015.

[6] O. M. Faltinsen, I. A. Lukovsky, and A. N. Timokha, "Resonant sloshing in an upright annular tank," Journal of Fluid Mechanics, vol. 804, pp. 608-645, 2016.
[7] A. Royon-Lebeaud, E. J. Hopfinger, and A. Cartellier, "Liquid sloshing and wave breaking in circular and square-base cylindrical containers," Journal of Fluid Mechanics, vol. 577, pp. 467494, 2007.

[8] E. J. Hopfinger and V. Baumbach, "Liquid sloshing in cylindrical fuel tanks," in Proceedings of the Progress in Propulsion Physics, pp. 279-292, Brussels, Belgium.

[9] I. A. Lukovsky and A. N. Timokha, "Multimodal method in sloshing," Journal of Matematical Sciences, vol. 18, no. 3, pp. 239253, 2015.

[10] S. Rebouillat and D. Liksonov, "Fluid-structure interaction in partially filled liquid containers: A comparative review of numerical approaches," Computers \& Fluids, vol. 39, no. 5, pp. 739-746, 2010.

[11] J. W. Miles, "Internally resonant surface waves in a circular cylinder," Journal of Fluid Mechanics, vol. 149, pp. 1-14, 1984.

[12] J. W. Miles, "Resonantly forced surface waves in a circular cylinder," Journal of Fluid Mechanics, vol. 149, pp. 15-31, 1984.

[13] I. P. Gavrilyuk, A. N. Timokha, and I. A. Lukovsky, "A multimodal approach to nonlinear sloshing in a circular cylindrical tank," Hybrid Methods in Engineering, vol. 2, no. 4, pp. 463-483, 2000.

[14] R. E. Hutton, "Fluid-particle during rotary sloshing," Journal of Applied Mechanics, Transactions ASME, vol. 31, pp. 145-153, 1964.

[15] V. Reclari, Hydrodynamics of orbital shaken bioreactors [Ph.D. thesis], Laboratoire de Machines Hydrauliques, Ecole Polytechnique Federale de Lausanne, 2013.

[16] W. Weheliye, M. Yianneskis, and A. Ducci, "On the fluid dynamics of shaken bioreactors-flow characterization and transition," AIChE Journal, vol. 59, no. 1, pp. 334-344, 2013.

[17] A. Ducci and W. H. Weheliye, "Orbitally shaken bioreactorsviscosity effects on flow characteristics," AIChE Journal, vol. 60, no. 11, pp. 3951-3968, 2014.

[18] M. Reclari, M. Dreyer, S. Tissot, D. Obreschkow, F. M. Wurm, and M. Farhat, "Surface wave dynamics in orbital shaken cylindrical containers," Physics of Fluids, vol. 26, no. 5, 2014.

[19] J. Bouvard, W. Herreman, and F. Moisy, "Mean mass transport in an orbitally shaken cylindrical container," Physical Review Fluids, vol. 2, no. 8, 2017.

[20] L. Prandtl, "Erzeugung von Zirkulationen beim Schütteln von Gefäßen," ZAMM - Zeitschrift für Angewandte Mathematik und Mechanik, vol. 29, no. 1-2, pp. 8-9, 1949.

[21] J. W. Miles and D. M. Henderson, "A note on interior vs. boundary-layer damping of surface waves in a circular cylinder," Journal of Fluid Mechanics, vol. 364, pp. 319-323, 1998.

[22] I. A. Raynovskyy and A. N. Timokha, "Damped steady-state resonant sloshing in a circular base container," Fluid Dynamics Research, vol. 50, no. 4, Article ID 045502, pp. 1-27, 2018.

[23] A. J. Faller, “The constant- $V$ vortex," Journal of Fluid Mechanics, vol. 434, pp. 167-180, 2001. 


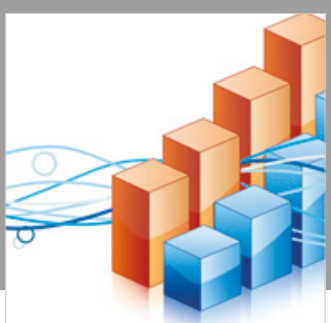

Advances in

Operations Research

\section{-n-m}
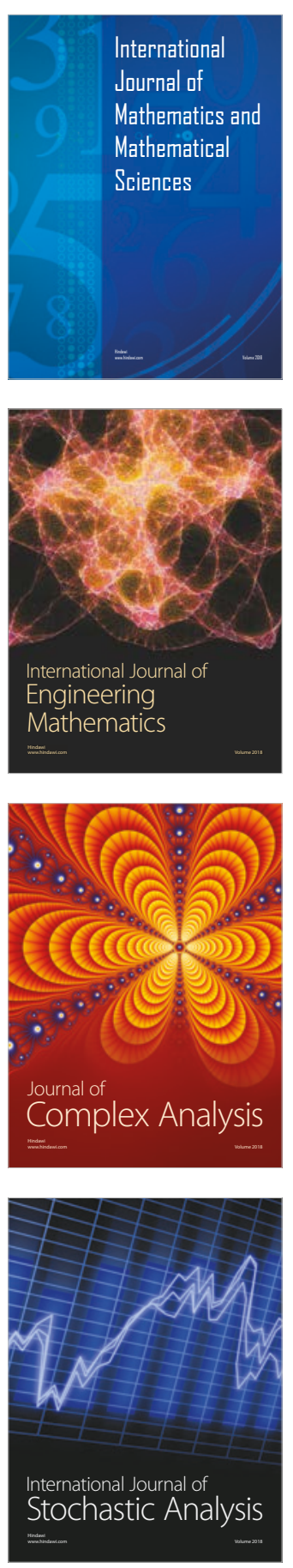
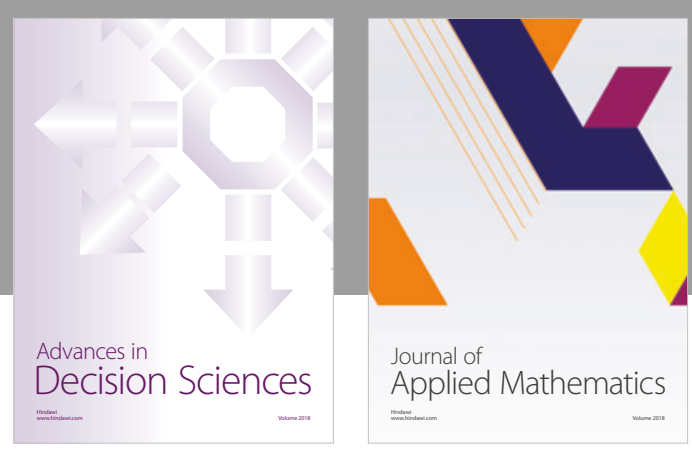

Journal of

Applied Mathematics
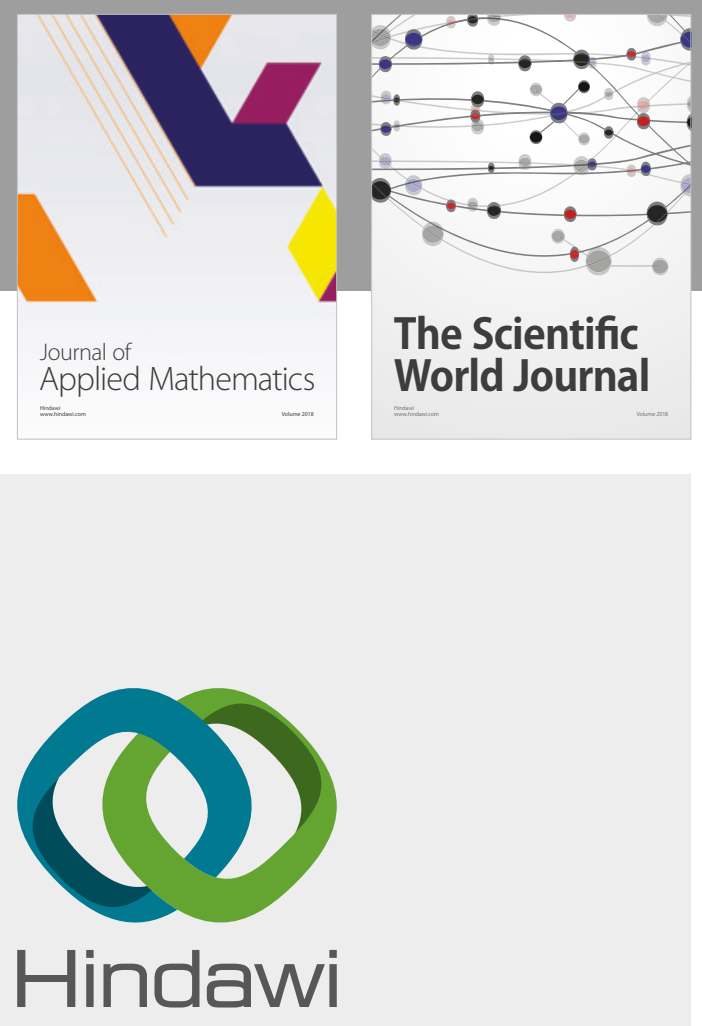

Submit your manuscripts at

www.hindawi.com

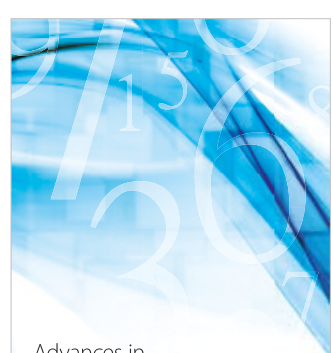

Advances in
Numerical Analysis
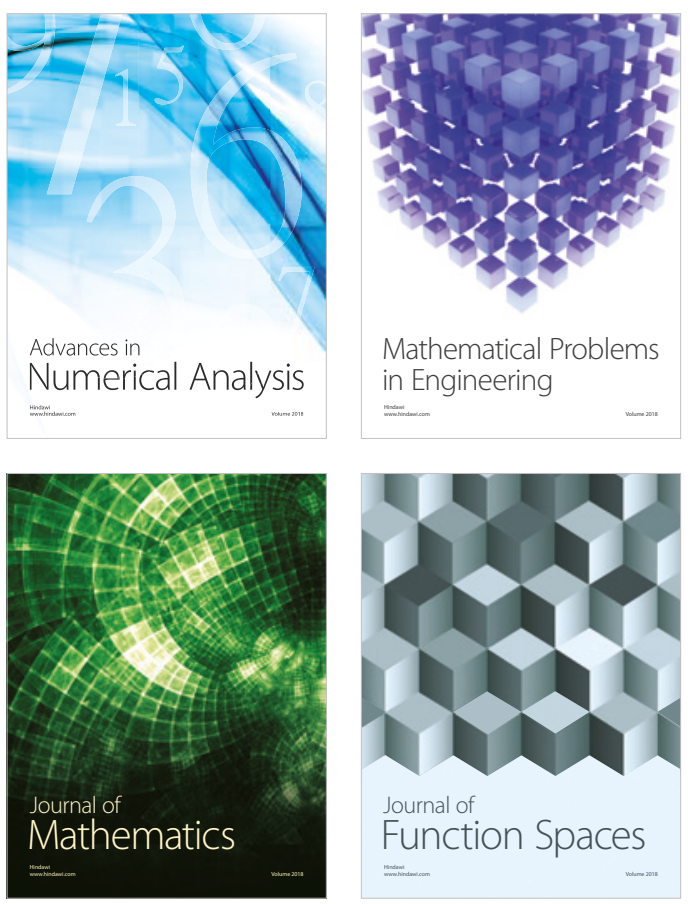

Mathematical Problems in Engineering

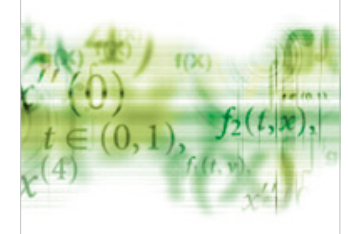

International Journal of

Differential Equations

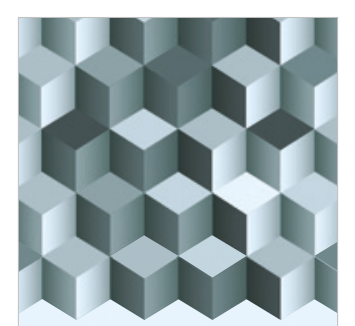

Journal of

Function Spaces

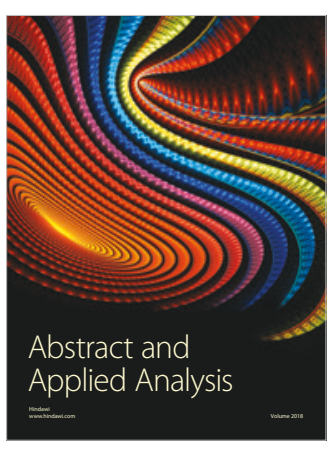

The Scientific

World Journal

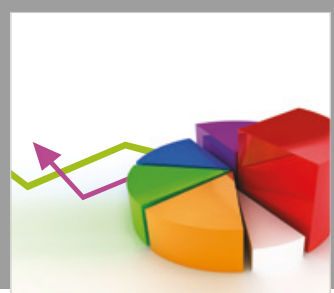

Journal of

Probability and Statistics
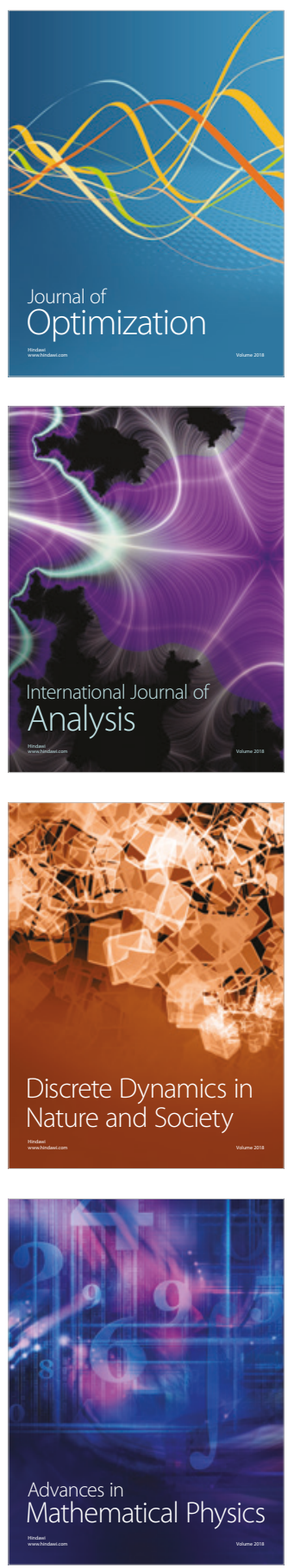